\title{
AN INTEGRATED MONITORING SYSTEM THROUGH 3D LASER SCANNER AND TRADITIONAL INSTRUMENTS FOR LOAD TEST ON ARCH BRIDGE
}

\author{
D. Pera, I. Ferrando* \\ Department of Civil, Chemical and Environmental Engineering, University of Genoa, via Montallegro 1, 16145 Genoa, Italy \\ (davide.pera, ilaria.ferrando)@edu.unige.it
}

\section{Commission}

KEY WORDS: Integrated monitoring system, load test, laser scanner, LVDT, masonry arch bridge

\begin{abstract}
:
The experimental campaign represents an example of how the careful design of the different test phases and the choice of the needed instrumentation are fundamental aspects to obtain a proper interpretation of the results, for future application on the real structures. Additionally, the present work represents a successful example of a combined monitoring system, integrating the traditional and innovative technical instrumentation for loading tests and geomatics survey techniques. The monitoring system has been designed with the aim of defining the load carrying capacity of a masonry arch bridge scaled model and to test the performances of a new retrofitting method. In particular, two different configuration have been considered: a first one with isolated arch and a second one with gravel fill on the arch.
\end{abstract}

\section{INTRODUCTION}

In many scientific fields, the experimental tests on reduced scale models are fundamental to understand the physical and mechanical phenomena and to simulate the behavior of a prototype. This approach is particularly convenient when the real scale simulation is not possible or it requires high costs. Besides these considerations, the repeatability of the tests, the possibility to study the different parameters' effect and to evaluate the variability of the results represent the main advantages of the employment of reduced scale models. Moreover, the tests on reduced scale models allow the a priori definition of the method and the needed technical instrumentation for data acquisition. Nevertheless, in order to obtain congruent results with the prototypes, it is crucial to examine the magnitudes that describe the physical phenomenon. In this context, the dimensional analysis is a key instrument to simplify the problem, reducing the number of variables. Lastly, the knowledge of the orders of magnitudes of the physical phenomena is likewise important in the choice of the instrumentation to be used, in terms of sensitivity and precision. The experimental campaign is a clear example of how the careful design of the different test phases, the choice of the needed instrumentation and the integration between different technologies to fill the mutual gaps are fundamental to obtain a proper interpretation of the results.

\section{STATE OF THE ART AND PROPOSED METHOD}

The number of masonry arch bridges still in service on the European railway is significant (about $35 \%$ overall) and the average time of construction dates back to more than 50 years ago. Consequently, a renovation of many of these structures is mandatory to stand the present rail traffic and to allow the transit of modern vehicles. The choice of the most appropriate maintenance and renovation technique should ensure the following aspects:

- substantial bearing capacity increasing;

- low invasiveness on the existing structures;
- limited traffic interruption;

- execution practicality;

- low cost.

Many of the typically employed reinforcement methods neglect these considerations. On one side, the retrofitting techniques aiming to block the formation of four plastic hinges, the characteristic collapse mechanism of arches, neglect the effect of the other components, this leading to a limited bridge's bearing capacity increase. On the other side, the techniques more effective on the maximum load increase are more invasive (e.g. concrete saddling), because they strongly modify the structure, with high costs in terms of time and execution (Brencich, 2007).

The presented experimental campaign was conducted to find a retrofitting technique to produce a significant increase of load carrying capacity, with a limited traffic interruption and a low invasiveness. Observing the behavior of real bridges in collapse conditions, it emerges that, among the other elements, the filling mainly contributes to the structure resistance. In particular, for structures with rise-to-span ratio higher than $1 / 3$, the presence of backfill reduces the span of the structural arch. The backfill is a portion of the fill located in the area over the bridge's piles, with strengthened mechanical characteristics, as Figure 1 shows.

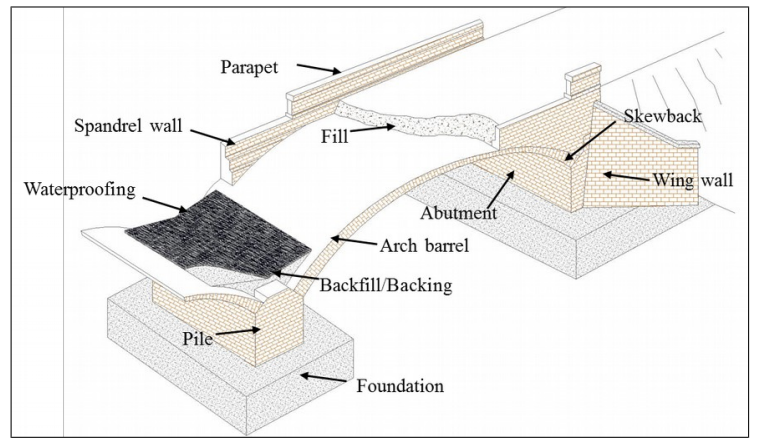

Figure 1. Elements of an arch bridge. 
To evaluate the effect of an increase in the mechanical characteristics of the fill, it was decided study the possibility to create a reinforced area near the backfill. The innovative method consists in the injection of binding material through the spandrel wall, without removing the fill, thus the traffic is not interrupted for a long time. The increased stiffness in the injection area induces an additional reduction of the arch span and, consequently, an increase of load carrying capacity. Figure 2 shows the effect of injection of binding material on the collapse mechanism of the arch.
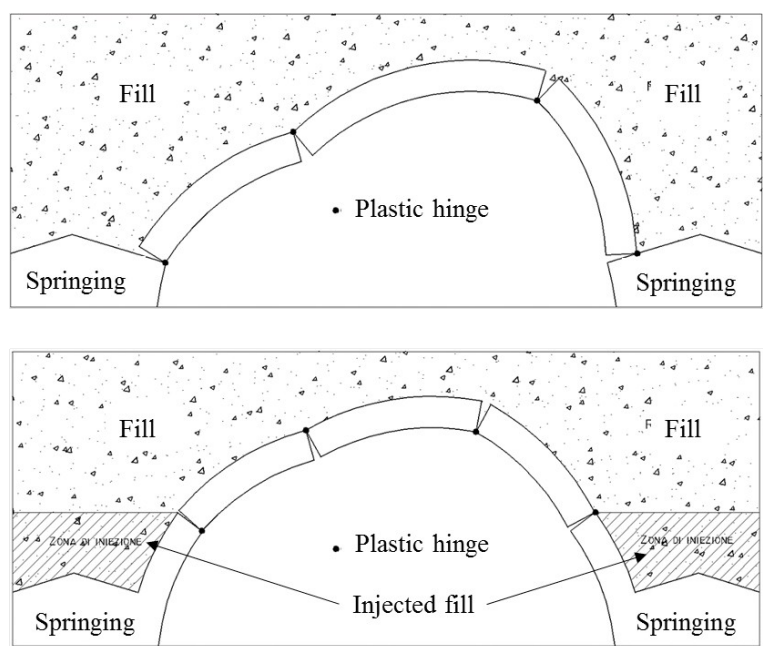

Figure 2. Collapse mechanism of an arch without (top) and with (bottom) injected fill.

Further details on the innovative method and the related analysis of such problem, which lie outside the present work, are given in Brencich and Pera (2016).

\section{EXPERIMENTAL CAMPAIGN}

A measurement campaign has been carried out on reduced scale models, in order to verify the effectiveness of the retrofitting method. The models, representing an internal portion of a real bridge, consist of a dry assemblage (without mortar) of aerated autoclaved concrete blocks. The skewback are made in concrete casted in place. The fill is made with gravel (non-cohesive material) and it is contained by Plexiglas ${ }^{\circledR}$ panels and a wooden structure supported by steel elements, as shown in Figure 3 .

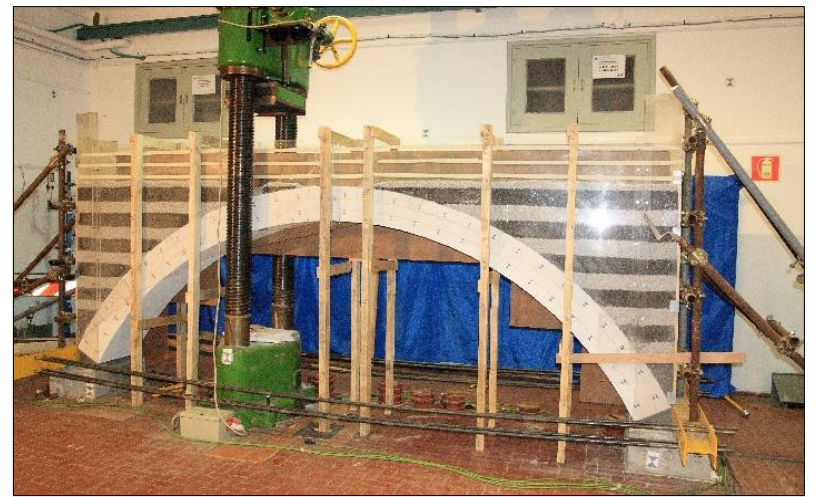

Figure 3. The arch model.

The model has been tested in several load tests in different setups, in order to obtain the load carrying capacity for:
- isolated arch;

- multi-span bridge model (with fill and deformable springing);

- $\quad$ single span bridge model (with fill and rigid springing);

- retrofitted multi-span bridge model ;

- retrofitted single span bridge model.

\subsection{Model realization and preliminary numerical analysis}

The reduced scale tests needed proper scaling rules in order to extend the results on real structures (prototypes). The adopted scaling rules descend from the dimensional analysis of the masonry bridges' static response problem and the Buckingham theorem application on it. As Riotto (2004) described, this approach reduces the number of parameters describing the problem, by means of expressing some of them as a function of the others.

The main characteristics of real bridges can be summarized as follows:

- typical span of Italian railway bridges: $12-18 \mathrm{~m}$;

- masonry strength: $10 \mathrm{MPa}$;

- medium density of bridges: $1 / 4$ arch (16.5) + 3/4 fill (17.5) $=16.75 \mathrm{kN} / \mathrm{m}^{3}$;

- non-cohesive fill material.

Whereas, the main characteristics of the models are:

- span: $4 \mathrm{~m}$;

- width: $0.45 \mathrm{~m}$

- cellular concrete strength: $2.4 \mathrm{MPa}$;

- medium density of model: $1 / 4$ arch (4.7) $+3 / 4$ fill (14) $=$ $11.67 \mathrm{kN} / \mathrm{m}^{3}$;

- non-cohesive fill material

These values allow to simulate the behavior of a $1 / 3$ geometric scaled model, and to scale the strength $(1 / 4)$ and the density $(1 / 1.5)$ too.

A schematic representation of the model is given in Figure 4; the green arrow identifies the load application point.

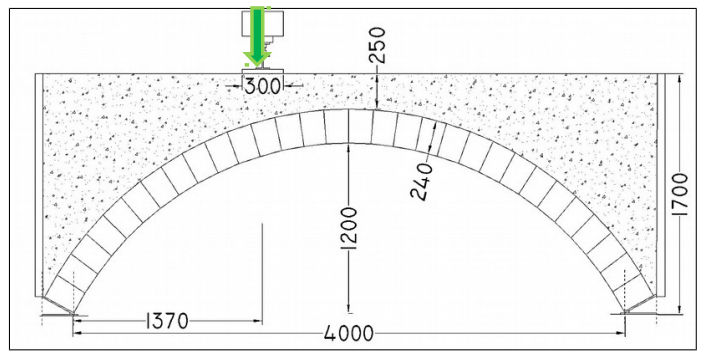

Figure 4. A scheme of the arch model.

In the preliminary phases, several numerical analyses have been performed, using a masonry bridges dedicated software, developed at Sheffield University. They allowed obtain a preliminary indication on the entity of magnitudes to be measured during the load tests, with particular attention to loads and displacements, and to choose the appropriate instruments to measure the different parameters with high accuracy.

The primary analyzed parameter was the model's maximum load. The load tests have been designed to overcome the model's limit load, imposing a displacement at $1 / 3$ of the model's span and measuring the corresponding force by means of load cells.

Figure 5 represents the deformed shape corresponding to the limit conditions of the model with fill. The numerical analysis outputs give useful indications on the collapse mechanism, showing the portions of the model experimenting the highest displacements. 


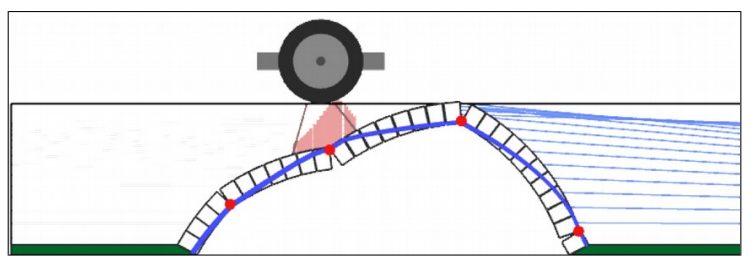

Figure 5. Numerical analysis output: models' deformed shape.

The red points in Figure 5 represent the plastic hinges positions; the red area indicates the load propagation in the fill, from the application point to the arch barrel.

As already stated, the fill plays a fundamental role in the bridges' behavior; in particular, it is widely known that the fill mechanical characteristics affect the dispersion angle, representing the inclination of load propagation. The entity and the characteristics of load distribution are still unclear, and they represent the primary cause of uncertainty on the collapse load estimation. Moreover, another important aspect to take into account is the deformability of the springing (steel element in Figure 3). The different stiffness of these elements generates pressures inside the fill, in the area near the skewback, which produces a passive pressure state in the fill, yielding an increased stability to the whole structure. The area affected by this effect is represented with blue lines in Figure 5.

\subsection{Employed instrumentation and measurement set-up}

To measure the displacements of the blocks near the plastic hinges, 11 LVDTs (Linear Variable Differential Transformers) were installed, as Figure 6 shows. The applied load is measured by load cell. To estimate the diffusion of load through the fill, pressure cells were installed along the arch extrados, in the area under the load application (red area in Figure 5). Lastly, pressure cells were also installed in the area near the skewbacks, where the passive pressure state is present.

Figure 6 reports a scheme of measurement set-up. The positions of LVDTs are depicted as blue arrows (representing the direction and the inclination of LVDTs), the pressure cells along the extrados are represented in red, and the black springs on the right side are the constraints, representing the lateral support deformability.

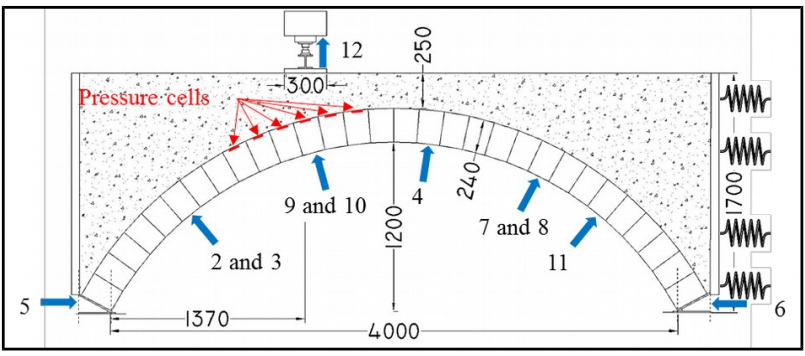

Figure 6. Scheme of measurement set-up.

A detailed description of the different instruments and on the operational choices are given in the next sections. These settings allowed to monitor the model during the load tests. The employment of both direct and remote, continuous and instantaneous measuring techniques allowed to overcome the limits of the traditional load test measuring systems, keeping an acceptable computational effort.
3.2.1 Load cell: The force due to the imposed incremental displacement was measured with a load cell, with different settings according to the arch configuration. Two different load cells were chosen, with different calibration, based on the maximum estimated load:

- for isolated arch: load cell with $25 \mathrm{kN}$ maximum load; calibrated from 0 to $10 \mathrm{kN}$;

- for arch with fill: load cell with $200 \mathrm{kN}$ maximum load; calibrated from 0 to $50 \mathrm{kN}$.

3.2.2 Pressure cells: Several pressure cells have been placed along the arch extrados, in order to obtain useful information on the load distribution through the fill. The main components of the pressure cell are:

- a flat rectangular metallic membrane, containing a fluid;

- a stem, containing the pressure transducers;

- a cable.

The measuring interval of pressure cells is from 0 to 7 bar.

Different elements, i.e. membrane shape and dimensions, cellterrain stiffness ratio, placement method and pressure measurement system, affect the quality of pressure measurements (Weiler and Kulhawy, 1982; Passalacqua et al., 1991; Schmertmann, 2005).

The choice of a commercial solution allowed not to analyze in details the different mentioned factors, to restrict the analysis to some characteristics and to control the calibration.

As already stated, one of the most influential aspects for measurements quality is the placement of the pressure cells. In order to obtain reliable pressure measurements on the arch surface, it is necessary to work with undisturbed terrain around the pressure cell. For this reason, a through hole was created in some blocks, to allow the placement of the stem and leave the membrane attached to the surface of the arch, as Figure 7a and $7 \mathrm{~b}$ show. Additionally, as Figure $7 \mathrm{c}$ depicts, a textile material (typical for geotechnical use) and a thin sand layer were placed to protect the cell from punching, which strongly affects the pressure measurement producing a localized load on the cell that increases the measured pressure, and can damage the pressure cell itself.

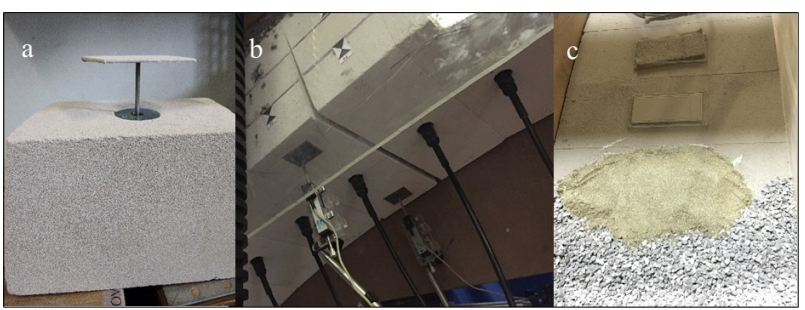

Figure 7. Pressure cell installation.

In order to obtain a correct interpretation of the results, the pressure cells were calibrated according to the load test conditions on the models.

3.2.3 Displacement sensor: As depicted in Figure 6, 11 LVDTs have measured the imposed displacement (LVDT 12, placed on the load beam) and the corresponding arch displacements (LVDTs 2-11, placed on the intrados of the blocks near the possible plastic hinges). Figure 8 represents a detail of a LVDT. 


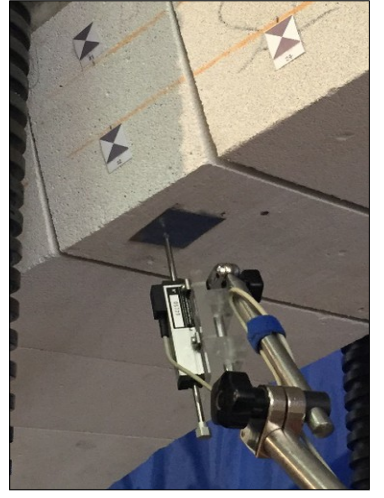

Figure 8. A detail of LVDT.

The main advantages in using this kind of sensors are the high accuracy and the possibility of continuously measuring the displacements; in fact, LVDTs have a nominal accuracy of $0.001 \mathrm{~mm}$ and can register the data with a frequency of $1 \mathrm{~Hz}$. On spite of these excellent qualities, this technique is quite limited in the information it gives; in fact, LVDTs measure only the movements of the blocks where they are placed. Additionally, the shape of the structure induces to place the sensors in orthogonal direction with respect to the blocks' surfaces, with the effect that LVDTs measure the radial movements of the blocks, thus it is not possible to estimate the roto-translation of the blocks. These limits can be easily overcome thanks to a careful placement planning and installation phase. Nevertheless, the measurement on radial direction is valid for low load levels; instead, in the large displacements field, which is reached increasing the load and approaching the maximum load, the block rotation causes the loss of orthogonality. To measure the blocks rotation, the number of LVDTs should be increased, this leading to a rise of cost in terms of data acquisition, management and elaboration, in addition to instrument cost.

3.2.4 Laser scanner: Laser scanner technology has been widely used to study real structures and describe their geometry, for both cultural heritage (Aita et al., 2017; Caroti et al., 2003) and civil engineering studies (Caroti et al., 2012; Visintini and Spangher, 2013).

The present study represents a novelty because the laser scanner has been used to survey a reduced scale model to study the applicability of an innovative retrofitting method, to be applied on real structures. The $\mathrm{Z}+\mathrm{F}$ Imager ${ }^{\circledR} 5006 \mathrm{~h}$ laser scanner was employed to overcome the limits of LVDTs in large displacements field and to estimate the inclination of the blocks with respect to the initial configuration, in parallel to LVDTs. The laser scanner is able to give a global 3D description of the model and to provide useful information on the rotation of blocks, which is helpful both for the displacements evaluation, to be integrated with LVDTs measures, and for the estimation of the forces migrating through the fill, measured with the pressure cells.

Figure 9 shows the experiment set-up, with an indication of the position of the laser scanner with respect to the model. The choice of a non-centered position of laser scanner is due to the presence of a camera in front of the arch. As described in more detail in the next section, the camera was used for continuous image acquisition during the load test, to be used in testing the applicability of photo-tracking and PIV (Particle Image Velocimetry) techniques.

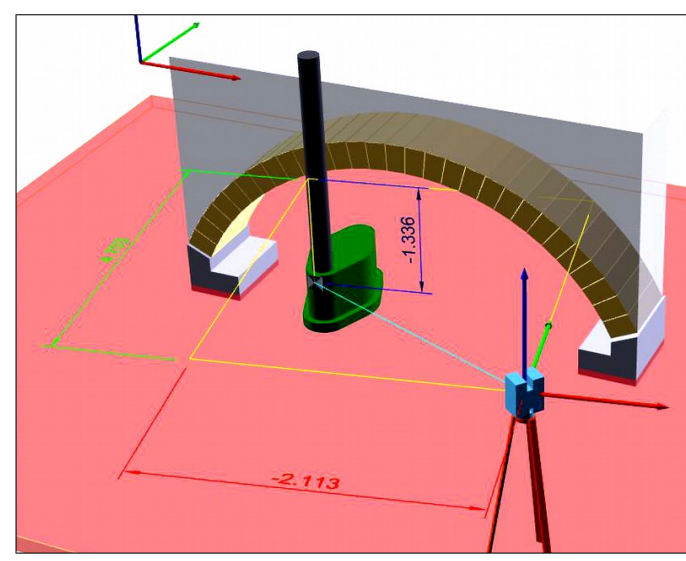

Figure 9. Experiment set-up: laser scanner position.

In the present case, the main advantages of using a laser scanner are the availability of spatial information for each point of the model, the quite limited need of additional instruments and the repeatability of the scanning in time, producing a monitoring of the arch. Among the disadvantages, the need to interrupt the load test during the scanning operation must be cited. This could affect the results due to the non-constant measured load at the same level of imposed displacement. Figure 10 depicts the load-displacement curve. The red and blue circles highlight the moments of laser scanner acquisition in different arch configurations; they correspond to an abrupt decrease of the measured load with a constant imposed displacement, due to the interruption of the load test.

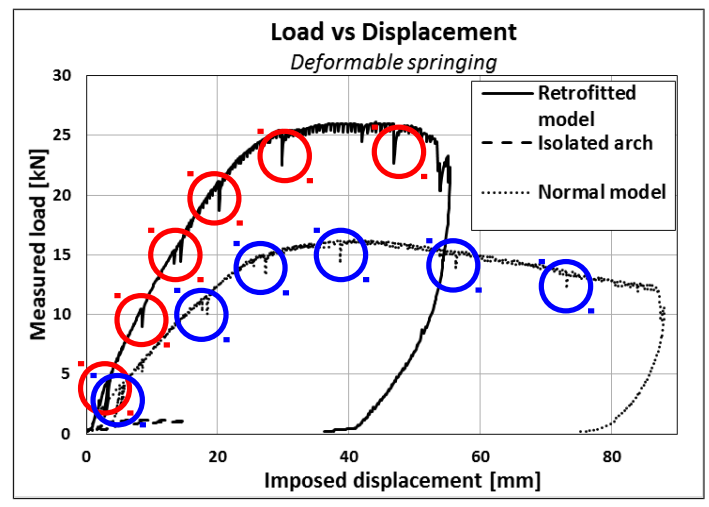

Figure 10. Load-displacement curve.

Red and blue circles highlight the laser scanner acquisitions.

The previous consideration led to these settings for laser scanner acquisition, in order to speed up the time of laser scanning and to keep the number of surveyed points low, for an agile elaboration phase:

- the acquisition was limited to the sector containing the arch;

- the superhigh resolution was chosen. It ensures a pixel pitch of about $3 \mathrm{~mm}$ at $10 \mathrm{~m}$ of distance and a scanning time of about 4 minutes for the chosen sector, considered compatible with the spatial and temporal scales involved in the load test;

- apart from the initial and the final scans, executed without any imposed displacement, the number of scans was planned in order not to have too many interruptions during the load test (maximum 7 interruptions).

In order to monitor the arch displacements in each load tests and to compare different arch configurations (corresponding to 
different load tests), a stable local reference frame was fixed on eight points, external to the arch. These points were used to orient all the scans of the different tested configurations, and they made possible the comparison of displacements of the surveyed points on the arch.

Two small targets, aligned along the vertical centerline of each block, identify the surveyed points of the arch, for which the coordinates have been extracted.

An example of laser scanner point cloud is given in Figure 11, for one of the phases of load test on isolated arch. The part of the arch near the load application point is hidden by the press, thus it does not appear in the point cloud.

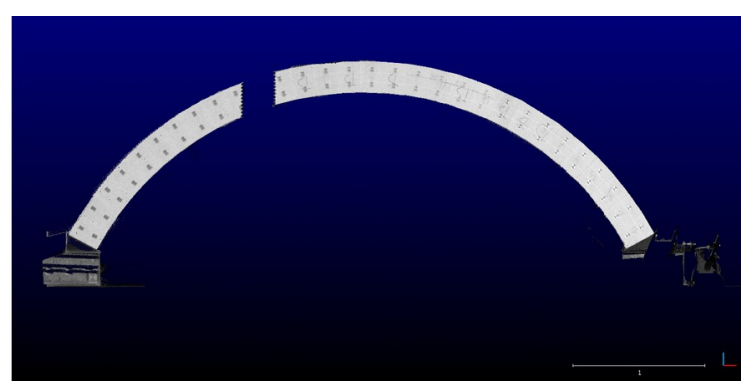

Figure 11. Laser scanner point cloud.

The mentioned laser scanner settings allowed to obtain the targets' coordinates with an accuracy of about $1 \mathrm{~mm}$.

Additional to the targets coordinates, the point cloud was used to realize an orthophoto for each phase of the load test. Thanks to the generated orthophotos, which will be presented in the following paragraph, it is possible to obtain the arch and blocks profiles using a CAD software, and to measure the inclination angle of each block.

Although the measure accuracy is not comparable with the one provided by LVDTs (nominal accuracy of $0.001 \mathrm{~mm}$ ) in the small displacement field, for large imposed displacements the laser scanner proved to have big potential. In fact, the rotations of the blocks make the LVDTs measures ineffective in describing the blocks' movements, while the measured coordinates and the orthophotos help in describing the global arrangement of the arch. Moreover, thanks to the orthophotos, it is possible to study the fill's deformations, even if only for some specific temporal moments.

This experimental campaign shows how using both the traditional and geomatics instruments gives more exhaustive information and allows a complete study for small and large displacements fields.

3.2.5 Digital camera: The survey techniques employing digital cameras are widely known and exploited. For example, photogrammetry provides a $3 \mathrm{D}$ representation of objects, using the principle of stereoscopy. With the diffusion of digital photography, photogrammetry has become widespread, also thanks to the use of ad hoc processing software.

PIV techniques (Particle Image Velocimetry), typically used in fluid dynamics experiments to measure the flow field, are able to give the instantaneous velocity field. PIV has been used for arch bridges (Callaway et al., 2012) for monitoring the movements of the fill during the load tests. The primary limit is the need of unconventional instruments, together with complex mathematical solvers, producing high costs and sophisticated experimental phases.

A digital camera was installed in front of the arch to test the photo-tracking technique. This method consists in the automatic tracking of the pixels in a sequence of images, thanks to the identification of common points (homologous points) in the different images, and allows to compute pixels' trajectories and velocities. Differently from PIV, this method can be applied using common commercial cameras, but it needs a fine calibration phase in order to reach the high quality standards requested by the computing algorithms for image processing. In fact, the processing software need accurate parameters, i.e. color saturation or grey scale level, to recognize the pixels with high precision. Moreover, the final accuracy of the trajectories and velocity field given by the technique should match with the one of the traditional load tests instruments.

During the load tests, images were acquired with a Canon reflex camera with the following characteristics and settings:

- sensor: $12 \mathrm{Mpx}$;

- focal length: $17 \mathrm{~mm}$;

- lens opening (iris): 4.0 ;

- exposure time: $0.1 \mathrm{~s}$;

- shutter speed: $0.1 \mathrm{~Hz}$

- stable, centered and fixed position for all the load tests, at a distance of $4 \mathrm{~m}$ from the arch.

The image processing has been performed through a tracking program developed in LabVIEW ${ }^{\circledR}$ environment, to provide the entity and the direction of pixels' displacements. The program works for both the arch and the fill pixels, but a deeper check on obtained precision is needed. This is possible comparing the photo tracking results with the ones coming from LVDTs and laser scanner. These aspects represent the further developments of the present work.

\section{RESULTS}

In the initial phase of each load test, load and un-load cycles were applied on the model, with a limited entity of loading, in order to eliminate possible constructive imperfections. The condition representing a stable configuration of the arch was defined based on the displacements sensors' measurements variability, with an accuracy of $0.001 \mathrm{~mm}$.

The continuous data acquisition allowed to obtain the loaddisplacement curve for the entire duration of each the load test, thus it was possible to compare the limit load, and the corresponding deformation, in different configurations. For example, Figure 12 reports the results for the isolated arch (dashed line), the arch with fill (dotted line) and the retrofitted model (line), highlighting the effectiveness of the injection of binding material, as the innovative retrofitting method proposes.

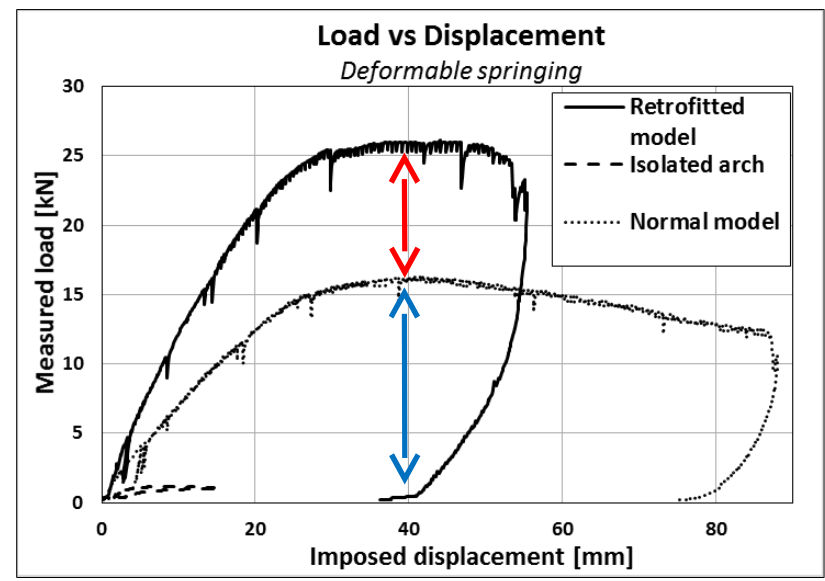

Figure 12. Load-displacement curve, showing the increase in measured load at fixed imposed displacements for the retrofitted model. 
For the configuration represented in Figure 12, the limit load increased from 1 to $16 \mathrm{kN}$ due to the fill (blue arrow), and from 16 to $26 \mathrm{kN}$ (red arrow), thanks to the selective binding material injection over the springings.

Another interesting result coming from the continuous data acquisition is the fill deformability in the area under the load application point, represented in Figure 13. Dashed and continuous lines represent the imposed displacement (directly applied on the fill) and the corresponding arch displacement, respectively. The different inclinations of the curves show the deformation absorbed by the fill.

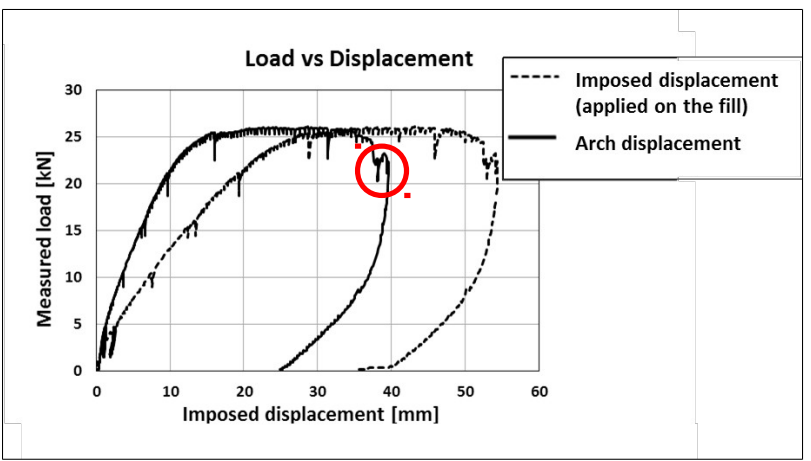

Figure 13. Curve of imposed and measured displacements.

The red circle in Figure 13 highlights an abrupt decrease in measured load, corresponding to a brittle fracture of one of the block, after the limit load has been reached. This kind of collapse behavior can be observed in a test in displacement control, where the behavior of the model can be monitored after the limit load, thanks to the continuous data registering by LVDTs. As already mentioned, the other abrupt decreases of load correspond to the moments when the load test was interrupted to perform the laser scanning.

The laser scanner scans have been used to describe the model behavior in the large displacement field, when the LVDTs are not exhaustive, due to block's roto-translations. The coordinates of the targets on the blocks, in the same reference frame for each performed load test, can be used in FEM (Finite Elements Model) analysis, to retrieve the displacements of the blocks, to be compared to the ones measured by LVDTs along radial direction, with the already mentioned limits in large displacements field. An example of comparison of blocks' displacements from the two methods is reported in Table 1: Table $1 \mathrm{a}$ and $1 \mathrm{~b}$ report the horizontal and vertical displacements of the elements on different blocks, respectively; Table 1c reports the corresponding radial displacements, as measured by LVDTs.

The negative sign indicates a left and down displacement in Table $1 \mathrm{a}$ and $1 \mathrm{~b}$; and a displacement toward the arch center in Table 1c.

From the data obtained by laser scans, the inclination angles of the blocks have been computed, in different load conditions and configurations.

\begin{tabular}{|l|l|c|c|c|c|c}
\hline \multirow{2}{*}{$1 \mathrm{c}$} & \multicolumn{5}{|c}{ I.ASFR SCANNER $\Delta \mathrm{x}[\mathrm{mm}$} \\
\cline { 3 - 8 } & & scan 1 & scan 2 & scan 3 & scan 4 & \\
\hline \hline \multirow{2}{*}{ Block 12 } & mark 21 & 0 & -1 & 2 & 3 & \\
\cline { 2 - 8 } & mark 22 & 0 & -1 & 2 & 6 & \\
\hline Block 17 & mark 32 & 0 & -1 & 2 & 5 & \\
\hline \multirow{2}{*}{ Block 22 } & mark 41 & 0 & 1 & 5 & 10 & \\
\cline { 2 - 8 } & mark 42 & 0 & -1 & 4 & 9 & \\
\hline
\end{tabular}

\begin{tabular}{|l|l||c|c|c|c|c}
\hline \multirow{2}{*}{1} & \multicolumn{5}{c}{ LASER SCANNFR $\Delta y[\mathrm{~mm}$} \\
\cline { 3 - 8 } & scan 1 & scan 2 & scan 3 & $\operatorname{scan} 4$ & \\
\hline \multirow{2}{*}{ Block 12 } & mark 21 & 0 & 2 & 4 & 9 & \\
\cline { 2 - 7 } & mark 22 & 0 & 2 & 6 & 10 & \\
\hline Block 17 & mark 32 & 0 & 1 & 2 & -2 & \\
\hline \multirow{2}{*}{ Block 22 } & mark 41 & 0 & 0 & -4 & -10 & \\
\cline { 2 - 7 } & mark 42 & 0 & 0 & -3 & -10 & \\
\hline
\end{tabular}

\begin{tabular}{|l|c|c|c|c|c}
\hline \multirow{2}{*}{1 1c } & \multicolumn{5}{|c}{ LVDT radial displacement [mm } \\
\cline { 2 - 6 } & $\operatorname{scan} 1$ & $\operatorname{scan} 2$ & $\operatorname{scan} 3$ & $\operatorname{scan} 4$ & \\
\hline Block 12 & 0 & -0.51 & -3 & -6.92 & \\
\hline Block 17 & 0 & 0.16 & 1.18 & 4.09 & \\
\hline Block 22 & 0 & 1.01 & 6.52 & 15.76 & \\
\hline
\end{tabular}

Table 1. Laser scanner (a and b) and LVDTs (c) displacements.

Additionally, the orthophotos have given geometric information (block inclinations and displacements) of all the arch elements. Figure 14 represents the initial (red) and final (light blue) position of a block, as retrieved from the orthophotos.

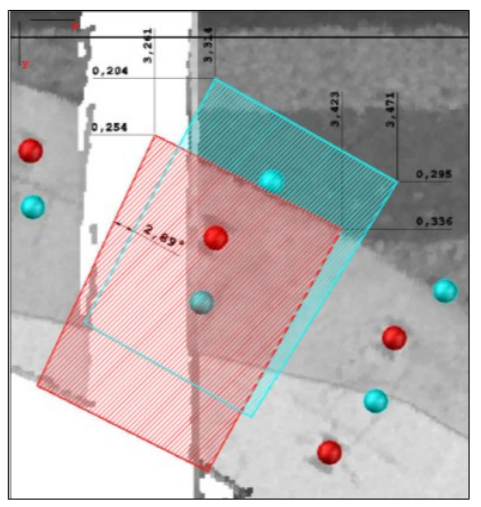

Figure 14. Blocks displacements from orthophotos.

The blocks' inclination angles have been used to compute the force migrating through the fill and reaching each block, following the scheme depicted in Figure 15, starting from the pressure measured by pressure cells.

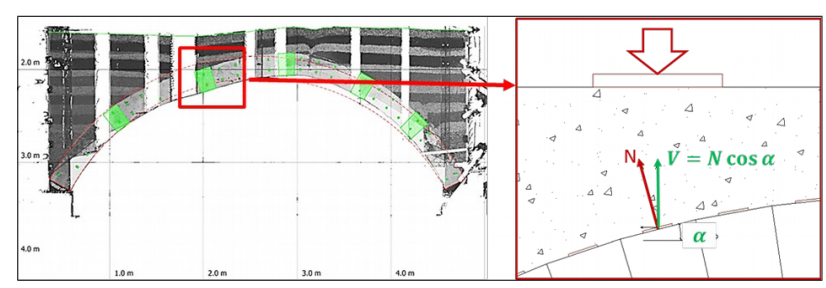

Figure 15. Method for vertical pressure (V) computation.

The measured pressure is orthogonal to the block surface $(\mathrm{N}$ in Figure 15), whereas the load is applied in vertical direction. To compare the action and reaction, the vertical direction has been chosen. Figure 16 represents an example of this computation. As Figure 16 shows, the lithostatic load and the load increase due to the imposed displacement give the total force on each block, along vertical direction. 


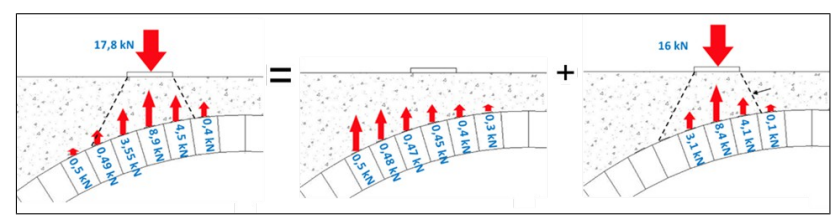

Figure 16. Force distribution on the blocks

The expected load distribution, as literature studies suggest, is represented in dashed lines in Figure 16. Thanks to the load tests, it was found that the load is distributed on larger surfaces if the fill has lower friction angle. The entity of this load is not homogeneous on the surface, but it is concentrated on the nearest block to the load application point.

Finally, the images, registered with a shutter speed of $0.1 \mathrm{~Hz}$, show the gradual deformation of the model.

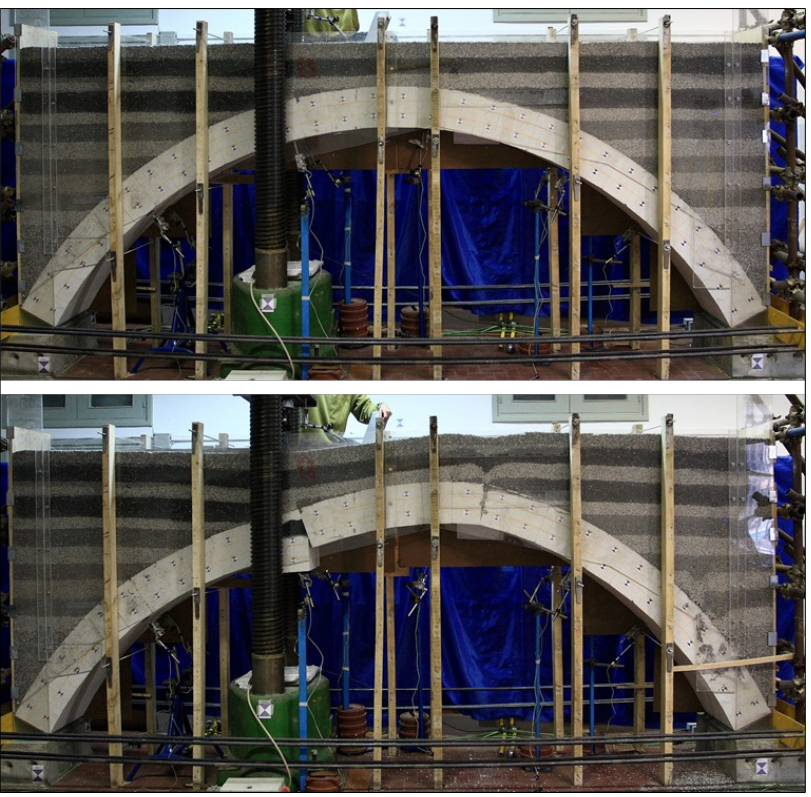

Figure 17. Gradual deformation of the model.

Additional to the fixed-camera images, some detail images were taken, in order to ascertain the contact area of the blocks and the progressive damage near the plastic hinges. Figure 18 represents two cracking modes: on the left a progressive "ductile" cracking, due to load increase; on the right a "brittle" cracking, which indicates the resistant portion of the block, highlighting the line of thrust position.

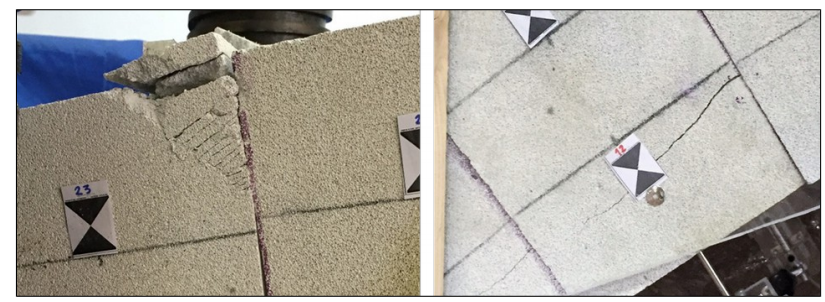

Figure 18. Cracking modes.

\section{CONCLUSIONS}

The presented experimental campaign represents an example of how a carefully planned measuring and monitoring system helps to obtain more accurate and complete information, also thanks to the employment of numerical models in the preliminary analysis of the problem.

The definition of the scale laws describing the similarity between the real bridges and the model led to the realization of the scaled model, properly scalded in both geometry and mechanical features.

The monitoring system has been designed with the aim of defining the load carrying capacity of the arch in different configurations.

The displacements have been directly and continuously measured with 11 Linear Variable Differential Transformers (LVDTs), with a nominal accuracy in the order of $0.001 \mathrm{~mm}$. Moreover, the need of measuring the load distribution through the fill led to the employment of pressure cells, placed between the blocks and the fill. To compare the imposed load and the pressure on the arch, the same vertical reference system for LVDTs and pressure cells was realized. To refer the pressure cells data, measured in the perpendicular direction with respect to the blocks surface, to the vertical direction, the inclination angles of the blocks were retrieved from laser scanner acquisition. The scans were used to generate orthophotos, which resulted extremely useful to describe the global behavior of the arch in the different load configurations.

The so realized monitoring system and the deriving products allowed to measure the single displacement of the blocks equipped with LVTDs, the roto-translations of all the blocks and to obtain the coordinates of interesting points on the blocks, to be used to derive the inclination angles.

Comparing the results between the two techniques, i.e. the traditional load test instrumentation and the alaser scanner, emerged that the LVDTs measures are more reliable when the deformations are small, whereas the displacement measured through the orthophotos, generated by laser scans, are more accurate when the conditions are closer to the limit load.

In fact, since the block is supposed to move in the axial direction, the high precision of LVDTs measures can represent the displacement; nevertheless, when a block experiments both translation and rotation, the LVDT is not able to follow its movements correctly.

On the contrary, the 3D laser scanner products are not able to reproduce the sub-millimetric displacements, but they are useful to describe the global behavior of the structure, even in case of big displacements.

For this reason, the experience shows how a good designed monitoring system, combining traditional load test instruments and a 3D laser scanner, can be employed to obtain a complete description of the displacements and to overcome the limits of the two techniques.

The integration of traditional and geomatics survey techniques allowed to limit the number of instruments tools and reduce time and processing costs. The use of the laser scanner has allowed a limited number of LVDTs, making not necessary to monitor both vertical and horizontal directions, to retrieve the block rotations.

In the future perspective of using the proposed retrofitting method on real structures, it is certainly worth considering a photographic monitoring. If the system provided enough precision, it could overcome several environmental and economic issues. Systems based on laser technologies are very costly, while the direct ones, with sensors in contact with the structure, can be difficult to install (e.g. for high viaducts) and to maintain. Of course, the system has to be properly developed, thoroughly evaluating the accuracy. 


\section{REFERENCES}

Aita D., Barsotti R., Bennati S., Caroti G., Piemonte A., 2017. 3-dimensional geometric survey and structural modelling of the Dome of Pisa cathedral. ISPRS Archives, vol. XLII-2/W3

Brencich A., 2007. Guide to controls and repairs of masonry bridges. Document developed for International Union of Railways (UIC)

Brencich A., Pera D., 2016. A new retrofitting technique for masonry bridges. ARCH'16 Conference, Wroclaw, Poland

Callaway P., Gilbert M., Smith C.C., 2012. Influence of backfill on the capacity of masonry arch bridges. Bridge Engineering, Volume 165, Issue BE3, pp. 147-158

Caroti G., De Falco A., 2003. Geometric survey for the structural assessment of the architectural heritage: the case of the Baptistery of S. Giovanni e Reparata in Lucca. ISPRS Archives, vol. XXXIV, pp. 111-116

Caroti G., Franconi A. Piemonte A., 2012. Metodologia di elaborazione dati laser scanner per la generazione di modelli utili al calcolo strutturale. Atti della $16^{\text {a }}$ Conferenza Nazionale ASITA, pp. 383-390

Repair and strengthening of Masonry Arch Bridges Background Report for Literature Review. Sustainable Bridges. 2007 Assessment for Future Traffic Demands and Longer Lives.

Passalacqua R., Berardi R., Cabella E., 1991. Experimental modeling of soil-foundation interaction compared with nemerical analyses. Computational methods and experimental measurements V, pp. 675-686. Ed C.M.P. \& Elsevier.

Riotto G., 2010. Analisi sperimentale del comportamento statico e dinamico dei ponti in muratura. PhD Thesis, University of Genoa

Schmertmann J.H., 2005. Stress diffusion experiment in sand. Journal of geotechnical and geoenvironmental engineering, vol. 131, ${ }^{\circ} 1$, ASCE, ISSN 1090-0241/20005/1-1-10

Visintini D., Spangher A., 2013. Rilevamento laser scanning, modello della superficie (DSM) e modello per il metodo agli elementi finiti (FEM) di una struttura. Atti della $17^{\mathrm{a}}$ Conferenza Nazionale ASITA, pp. 1287-1294

Weiler W.A., Kulhawy F.H., 1982. Factors affecting stress cell measurements in soil. Proc. Paper 17553 GT12, pp 1529-1548

CloudCompare (version 2.6) [GPL software]. (2016). Retrieved from http://www.cloudcompare.org/

Z+F Imager ${ }^{\circledR}$ 5006h User Manual

Z+F LaserControl ver. 8.1 User Manual 